\title{
Fast ion JET diagnostics: confinement and losses
}

\author{
V.G. Kiptily, ${ }^{1}$ D. Borba, ${ }^{2}$ F.E.Cecil,${ }^{3}$ M. Cecconello, ${ }^{4}$ D. Darrow,${ }^{5}$ \\ V. Goloborod'ko, ${ }^{6,7}$ K. Hill, ${ }^{5}$ T. Johnson, ${ }^{8}$ A. Murari, ${ }^{9}$ F. Nabais, ${ }^{2}$ \\ S.D. Pinches, ${ }^{1}$ M. Reich, ${ }^{10}$ S.E. Sharapov, ${ }^{1}$ V. Yavorskij, ${ }^{6,7}$ \\ I.N. Chugunov, ${ }^{11}$ D.B. Gin, ${ }^{11}$ G. Gorini, ${ }^{12}$ A.E. Shevelev, ${ }^{11}$ D.B. Syme, ${ }^{1}$ \\ V. Zoita ${ }^{13}$ and JET-EFDA contributors ${ }^{14}$ \\ JET-EFDA, Culham Science Centre, OX14 3DB, Abingdon, UK \\ ${ }^{1}$ Euratom / UKAEA Fusion Association, Culham Science Centre, Abingdon, Oxon, United Kingdom \\ ${ }^{2}$ Euratom/IST Fusion Association, Centro de Fusão Nuclear, 1049-001 Lisboa, Portugal \\ ${ }^{3}$ Colorado School of Mines, Golden, CO, USA \\ ${ }^{4}$ Association EURATOM -VR, Royal Institute of Technology KTH, Stockholm, Sweden \\ ${ }^{5}$ Princeton Plasma Physics Lab, Princeton NJ, USA \\ ${ }^{6}$ Euratom/OEAW Association, Institute for Theoretical Physics, University of Innsbruck, Austria \\ ${ }^{7}$ Institute for Nuclear Research, Kiev, Ukraine \\ ${ }^{8}$ Euratom -VR Association, Alfvén Laboratory, Royal Institute of Technology, 10044 Stockholm, \\ Sweden \\ ${ }^{9}$ Consorzio RFX - Associazione Euratom-Enea sulla Fusione, I-35127 Padova, Italy \\ ${ }^{10}$ Euratom / MPI für Plasmaphysik Association, Garching, Germany \\ ${ }^{11}$ Ioffe Physico-Thechnical Institute, 194021 St. Petersburg, Russia \\ ${ }^{12}$ Istituto di Fisica del Plasma, EURATOM-ENEA-CNR Association, Milan, Italy \\ ${ }^{13}$ Euratom-MedC Association, National Institute for Laser, Plasma and Radiation Physics, Bucharest, \\ Romania \\ ${ }^{14}$ See the Appendix of M.L.Watkins et al., Fusion Energy 2006 (Proc. 21st Int. Conf. Chengdu, 2006) \\ IAEA, (2006)
}

\begin{abstract}
A study of magnetically confined fast ions in tokamaks plays an important role in burning plasma research. To reach ignition and steady burning of a reactor plasma an adequate confinement of energetic ions produced by NBI heating, accelerated with ICRF and born in fusion reactions is essential to provide efficient heating of the bulk plasma. Thus, investigation of the fast ion behaviour is an immediate task for present-day large machines, such as JET, in order to understand the main mechanisms of slowing down, redistribution and losses, and to develop optimal plasma scenarios. Today's JET has an enhanced suite of fast ion diagnostics both of confined and lost ions that enable to significantly contribute to this important area of research. Fast ion populations of $p, d, t,{ }^{3} \mathrm{He}$ and ${ }^{4} \mathrm{He}$, made with ICRF, NBI, and fusion reactions have been investigated in experiments on JET with sophisticated diagnostics in conventional and shear-reversed plasmas, exploring a wide range of effects. This paper will introduce to the JET fast-ion diagnostic techniques and will give an overview of recent observations. A synergy of the unique diagnostic set was utilised in JET, and studies of the response of fast ions to MHD modes (e.g. tornado modes, sawtooth crashes), fast ${ }^{3} \mathrm{He}$-ions behaviour in shearreversed plasmas are impressive examples of that. Some results on fast ion losses in JET experiments with various levels of the toroidal field ripple will be demonstrated.
\end{abstract}

Keywords: tokamak, burning plasmas, fast ions, diagnostics

PACS: $52.55 . \mathrm{Fa}$, 52.55.Pi, 52.70.La 
The nuclear reaction $D(T, n)^{4} H e$ between deuterium and tritium is the most promising source of energy in a thermonuclear fusion reactor. The power for the selfsustained $D T$-plasma burn is provided by the $\alpha$ particles $\left({ }^{4} \mathrm{He}\right.$-ions), which are born with an average energy of $3.5 \mathrm{MeV}$ and transfer the energy to the thermal plasma during their slowing down. To reach ignition and steady burning of a reactor plasma an adequate confinement of $\alpha$ particles and energetic ions produced by NBI heating, accelerated with ICRF is essential to provide efficient heating of the bulk plasma. Thus, investigation of the fast ion behaviour is an immediate task for present-day large machines, such as JET, in order to understand the main mechanisms of slowing down, redistribution and losses, and to develop optimal plasma scenarios.

Nuclear reaction $\gamma$-ray diagnosis is one of the important techniques used on the JET tokamak for studying confined fast ions [1-5]. The intense $\gamma$-ray emission is produced in JET plasmas when fast ions (ICRF-driven ions, fusion products, NBI-injected ions) react either with fuel ions or with the main plasma impurities such as carbon and beryllium. Gamma-ray energy spectra are recorded with collimated spectrometers, while the $\gamma$-ray emission spatial 2D-profiles are measured with the JET neutron/gamma profile monitor $[5,6]$. Together, these provide information on the spatial distribution of fast ions and fast ion tail-temperature.

On JET, the $\alpha$-particle diagnostic technique is based on the nuclear reaction ${ }^{9} \mathrm{Be}(\alpha, n \gamma){ }^{12} \mathrm{C}$ between confined $\alpha$-particles and the beryllium impurity typically present in the plasma. The $\gamma$-radiation due to the reaction ${ }^{9} B e(\alpha, n \gamma){ }^{12} C$ has been observed for the first time in JET experiments with the third harmonic ICRF heating of ${ }^{4} \mathrm{He}$ beam ions in a ${ }^{4} \mathrm{He}$ plasma $[3,5,7]$ and in $D$-plasmas with short $T$-NBI blips for $\alpha$-particles [4].

During recent experiments, JET $\gamma$-ray diagnostics provided some of the most interesting results on fast-ion and $\alpha$-particle physics [4,5]. These experiments on JET showed that the diagnostics work well, but a further improvement of the diagnostics is needed for the measurements in the high neutron yield $D D$ - and $D T$-discharges to demonstrate the capabilities of $\gamma$-ray diagnostics for burning plasma physics in future reactors.

Recently, two fast ion loss diagnostic devices we have been installed. These consist of a set of thin Faraday foil collectors and a scintillator probe. Thin Faraday foil detectors, in which ions that are lost from a fusion plasma are detected as current to ground in a metallic foil near the plasma boundary, have been used to investigate ion losses on NSTX, DIII-D and JET [8]. Similarly, scintillation detectors have been widely employed to study ion losses on TFTR, NSTX, and other machines. The present devices are intended to study lost ions in general and $D T$-fusion product alpha particles in particular during the upcoming JET campaigns. The design and construction of a fast ion loss diagnostic for JET may have application to the International Thermonuclear Experimental Reactor (ITER). 


\section{EXPERIMENTAL EQUIPMENT}

\section{Gamma-ray spectrometers}

Gamma-ray energy spectra are measured on JET with three independent devices, one with a quasi-tangential, and two with vertical line of sight, through the plasma centre. Two of these devices are a calibrated bismuth germanate (BGO) scintillation detectors with a diameter of $75 \mathrm{~mm}$ and a height of $75 \mathrm{~mm}$. One of them is located in a well-shielded bunker, which views the plasma tangentially. In order to reduce the neutron flux and the gamma-ray background, the front collimator is filled to a depth of $500 \mathrm{~mm}$ with polythene. Behind the scintillation detector, there is an additional 500 $\mathrm{mm}$ long dump of polythene and a 1000-mm long steel plug. The detector's line of sight lies in a horizontal plane about $30 \mathrm{~cm}$ below the plasma magnetic axis. Another BGO-spectrometer is viewing the plasma vertically through the centre $(\mathrm{R} \approx 2.95 \mathrm{~m})$. The $\gamma$-ray spectra are continuously recorded in all JET discharges over the energy range $1-28 \mathrm{MeV}$, with energy resolution of about $4 \%$ at $10 \mathrm{MeV}$. The third device for the gamma-ray energy spectrum measurements is a $\mathrm{NaI}(\mathrm{Tl})$ scintillation detector with a diameter of $125 \mathrm{~mm}$ and a height of $125 \mathrm{~mm}$, viewing the plasma vertically through the centre $(\mathrm{R} \approx 2.95 \mathrm{~m})$.

For identification of the fast ions, which exist in the plasma and produce the observed $\gamma$-ray emission, and in order to assess the effective tail temperatures of these fast ions, the $\gamma$-ray spectrum modelling code, GAMMOD [3] is used. This code is based on the known nuclear reaction cross-sections and it contains information on about hundred $\gamma$-ray transitions in the final nuclei of the low- $\mathrm{Z}$ impurity reactions. It also includes the $\gamma$-ray response function of the BGO-spectrometer. A Maxwellian energy distribution is used to describe the line-of-sight averaged tail of ICRHaccelerated ions. The GAMMOD code analysis gives the effective tail temperatures, the fast ion concentrations and the contribution to the neutron yield from the fast particle-induced reactions.

Measurements in high performance discharges with $\mathrm{NaI}(\mathrm{Tl})$ and $\mathrm{BGO}$ detectors showed the necessity to upgrade the data acquisition system (DAQ) in order to improve the energy and temporal resolution of the measurements in high performance $d t$ - and $d d$-discharges. Recently, a modern DAQ system for $\gamma$-ray diagnostics based on PCI transient recorder was installed. It is used for digitising the incoming signal at a sampling rate of $25 \mathrm{MHz}$ with an amplitude resolution of 14-bit at maximum average pulse rate up to $1 \mathrm{MHz}$ [9]. For a precise pulse height analysis (PHA) at high counting rates, sophisticated software has been developed. It allows avoidance of the pile-up effect and gain instability, which lead to a distortion of $\gamma$-ray spectra at high-count rates with fast rate variations. The main limitation for further improving of counting rate capabilities with $\mathrm{NaI}(\mathrm{Tl})$ and $\mathrm{BGO}$-detectors is the rather long scintillation decaytimes, of $250 \mathrm{~ns}$ and $300 \mathrm{~ns}$, respectively. It is planned to replace the existing detectors by modern fast high- $\mathrm{Z}$ heavy scintillators $\mathrm{LaBr}_{3}(\mathrm{Ce})$, known as "BriLanCe" and $\mathrm{Lu}_{1.8} \mathrm{Y}_{0.2} \mathrm{SiO} 5(\mathrm{Ce})$ - "LYSO". These new scintillators have short decay times, of 
$16 \mathrm{~ns}$ and $40 \mathrm{~ns}$, and high photons yields. Their striking properties open a possibility to extend the counting rate limit beyond the $5 \mathrm{MHz}$, and at the same time to improve the energy resolution for $\gamma$-ray spectrometry in the range $2-30 \mathrm{MeV}$.

\section{Gamma-ray imaging of fast ions}

Spatial profiles of the $\gamma$-ray emission in the energy range $\mathrm{E}_{\gamma}>1 \mathrm{MeV}$ have been measured in JET using the Gamma Cameras (Fig.1), which have ten horizontal and nine vertical collimated lines of sight $[5,6]$. Each collimator corresponds to a poloidalviewing extent at the centre of plasma of about $10 \mathrm{~cm}$. The detectors array is comprised of $19 \mathrm{CsI}(\mathrm{Tl})$ photo-diodes $(10 \mathrm{~mm} \times 10 \mathrm{~mm} \times 15 \mathrm{~mm})$. The data acquisition system accommodates the $\gamma$-ray count-rate measurements in four independently adjustable energy windows. This allows allocating $\gamma$-ray peaks for a given fast ion population in specific windows to count them separately. The MonteCarlo calculations of the detector response function show that the size of the CsI(Tl) scintillator is suitable for the $\gamma$-ray measurements in the $\mathrm{MeV}$-range. It is found that a noticeable contribution to the $\gamma$-ray spectrum with energy $E_{\gamma} \gg 2 m_{e} c^{2}$ is coming from the so-called double - and single -escape peaks, which is resulting from the high probability of electron-positron pair generation in the detector. The double-escape peak efficiency of this detector is about $3 \%$ that in this case is sufficient for reliable counting.

Experimental data obtained for the 19 lines of sight allows tomographic reconstruction for deriving the local gamma-ray emissivity in a poloidal cross-section. In the tomographic reconstructions the full geometry of the collimators and detector efficiencies are taken into account, but small effects of attenuation and scattering of the $\gamma$-rays are neglected. It is assumed that the distribution of the low-Z impurities is uniform in the plasma core (a region, where $50 \%$ of neutrons are produced) as confirmed by atomic spectroscopy measurements. For the tomographic reconstruction a constrained optimisation method [10] is used, which was successfully applied earlier to soft x-ray, bolometer and $\gamma$-ray measurements on JET [6].

\section{Lost ions diagnostics}

The Faraday cup array detects the current of fast ions at multiple poloidal locations, with a dynamic range from $10 \mathrm{nA} / \mathrm{cm}^{2}$ to $10 \mathrm{~mA}^{2}$ at a temporal resolution of $1 \mathrm{~ms}$. The detectable range of $\alpha$-particle energies is about $1-5 \mathrm{MeV}$. The energy resolution for $3.5 \mathrm{MeV} \alpha$ particles is estimated to be about $15 \%-50 \%$. The array consists of nine detectors spread over five poloidal locations (Z) between $22 \mathrm{~cm}$ and $80 \mathrm{~cm}$ below the midplane. Radially, the detectors are equally spaced on three locations between 25 and $85 \mathrm{~mm}$ behind the adjacent poloidal limiter. Each detector consists of at least four $75 \times 25 \mathrm{~mm}^{2} \mathrm{Ni}$ foils $(2.5 \mu \mathrm{m}$ in eight of the detectors and $1.0 \mu \mathrm{m}$ in the ninth), which are separated by insulating mica foils. Depending on its energy, a particle can pass through a certain number of foils before it is stopped in one foil, thus causing a current signal. The detection of the temporal evolution of the current signals in all foils in the 
radially and poloidally distributed detectors will allow a map of particle energies at different locations.

The scintillator probe allows the detection of ions with a pitch angle between $30^{\circ}$ and $85^{\circ}$ (5\% resolution) and a gyroradius between 30 and $140 \mathrm{~mm}$ (15\% resolution). It is located about $28 \mathrm{~cm}$ below the midplane. The underlying principle of scintillator measurements is the emission of light by a scintillating material after a particle strikes this material. Selection criteria for the particles that hit the scintillator are introduced by using a set of collimators within the magnetic field of JET. An optical arrangement within the scintillator probe is used to transfer the light emitted by the scintillator towards a charge-coupled device CCD) camera and a photomultiplier array through a coherent fibre bundle.

\section{DIAGNOSIS OF FAST IONS IN JET}

\section{Alpha-particle confinement and losses}

In the JET experiment with the $3^{\text {rd }}$ harmonic ICRH heating of a ${ }^{4} \mathrm{He}$ neutral beam $\left(\omega=3 \omega_{4 \mathrm{He}}\right)$ in a ${ }^{4} \mathrm{He}$ plasma [7], the $\gamma$-radiation due to the reaction ${ }^{9} \mathrm{Be}(\alpha, n \gamma){ }^{12} \mathrm{C}$ has been observed for the first time [3,5,7]. It was found that the ICRH waves accelerate the ${ }^{4} \mathrm{He}$ beam ions up to the energies in excess of $2 \mathrm{MeV}$. The presence of the 4.44$\mathrm{MeV}$ peak in $\gamma$-ray spectra is evidence for the existence of alphas with energies that exceed $1.7 \mathrm{MeV}$. These measurements also indicate a surprisingly high level of the $\gamma-$ ray emission from the reaction ${ }^{12} C(d, p \gamma)^{13} C$ meaning that some ICRH power was absorbed by deuterons at the third harmonic $D$-resonance, which coincides with the $3^{\text {rd }}$ harmonic ${ }^{4} \mathrm{He}$-resonance. The fast $D$-ions give rise to $3.09-\mathrm{MeV} \gamma$-rays from the reaction ${ }^{12} C(d, p \gamma){ }^{13} C$. A peak at $3.09 \mathrm{MeV}$ in recorded spectra reflects the presence in the plasma of fast $D$-ions with energies exceeding $0.5 \mathrm{MeV}$.

In these experiments the relaxation of the distribution functions of fast ${ }^{4} \mathrm{He}$-ions, accelerated with $3^{\text {rd }}$ harmonic ICRH of ${ }^{4} \mathrm{He}$-beam and D-minority ions, accelerated due to the parasitic ICRH absorption have been simultaneously studied during slowing down in ${ }^{4} \mathrm{He}$-plasma (2.2T/2MA, $51 \mathrm{MHz}$ ). The $\gamma$-ray decay-time for both ions was measured during notches in the ICRH power when the accelerated ion source was strongly reduced. The $\gamma$-ray spectra with $100-\mathrm{ms}$ integration time were recorded by means of a NaI-detector, which observes the plasma core through the vertical collimator. The data is obtained in discharges with monotonic q-profiles only. A clear correlation $\tau_{\gamma}^{H e} \sim 0.5 \tau_{\gamma}^{D}$ is observed. This is the first direct confirmation of the Spitzer slowing down dependence for different types of ions. An effect of the $\mathrm{A} / \mathrm{Z}^{2}$-factor for the classical slowing-down time is observed in these experiments.

In the Gamma Cameras, special energy windows, containing $3.09 \mathrm{MeV}$ and 4.44 $\mathrm{MeV}$ peaks with their single and double escape satellites, were set up to measure the spatial profiles of the $\gamma$-ray emission from $\mathrm{D}$ - and ${ }^{4} \mathrm{He}$-ions. The ability to separate $\gamma$ rays with the Gamma Cameras from different energy bands has allowed the study of 
the fast ion behaviour in an advanced regime plasma discharge with Alfvén cascades and sawtooth instabilities [5].

The first $\gamma$-ray measurements of fusion-born $\alpha$ particles were carried out in JET 'trace tritium' discharges [4], i.e. in deuterium plasmas with a small population of tritium Neutral Beam Injection (NBI) fast ions. The $\gamma$-ray emission from the nuclear reaction ${ }^{9} \mathrm{Be}(\alpha, n \gamma)^{12} \mathrm{C}$ is used to measure changes in the density of the fast $\alpha$ particles with energy $E_{\alpha}>1.7 \mathrm{MeV}$ in the post-NBI period. It was demonstrated in these experiments how the $\gamma$-ray spectrometry of the interaction between $\alpha$ 's and Be impurity in plasmas could be used in future magnetic fusion machines to obtain essential information on the slowing down and confinement of the fast $\alpha$-particles.

Another possibility to study fusion $\alpha$-particles is the $D\left({ }^{3} \mathrm{He}, p\right)^{4} \mathrm{He}$ reaction, which yields $3.7-\mathrm{MeV}$ alphas and $15-\mathrm{MeV}$ protons. As a rule $D_{-}^{3} \mathrm{He}$ experiments are carried out with the ICRF heating of the ${ }^{3} \mathrm{He}$-minority $\left(\omega=\omega_{3 \mathrm{He}}\right)$ in $D D$-plasmas. In the recent $D-{ }^{3} \mathrm{He}$ JET experiments $\alpha$-particle confinement was studied, and for the first time $\alpha$ particle losses have been measured. The reaction $D\left({ }^{3} \mathrm{He}, \gamma\right)^{5} \mathrm{Li}$, which is a weak branch $\left(10^{-5}\right)$ of the fusion reaction $D\left({ }^{3} \mathrm{He}, p\right)^{4} \mathrm{He}$ giving rise to fusion alphas, was identified in the plasma by $\gamma$-ray spectrometers. Nuclear reactions with main impurities, ${ }^{12} \mathrm{C}\left({ }^{3} \mathrm{He}, \mathrm{p} \gamma\right){ }^{14} \mathrm{~N},{ }^{9} \mathrm{Be}\left({ }^{3} \mathrm{He}, n \gamma\right){ }^{11} \mathrm{C}$ and ${ }^{9} \mathrm{Be}\left({ }^{3} \mathrm{He}, \mathrm{p} \gamma\right){ }^{11} \mathrm{~B}$, have been detected as well. The spectrum analysis with GAMMOD code shows that the energetic ${ }^{3} \mathrm{He}$-tail has an effective temperature in excess of $300 \mathrm{keV}$. Gamma-ray images of energetic ${ }^{3} \mathrm{He}$-ions $\left(\mathrm{E}_{3 \mathrm{He}}>0.9 \mathrm{MeV}\right)$ were recorded with $2-\mathrm{D} \gamma$-ray cameras.

Fast ion losses were studied with the scintillator probe. Distribution of fusion $\alpha-$ particles born in the plasma with this type of heating is rather anisotropic, and has a broad energy spectrum. According to the calculated $\alpha$-particle spectrum, most of alphas in the plasma have energy in range of $2-5.5 \mathrm{MeV}$, and that is consistent with energy range of maximum losses. The modelling of orbits of the lost $\alpha$-particles shows that most of energetic ions detected by the probe originates from the plasma region, which is coincided with the hot spot in the measured $\gamma$-ray emission profile. Thus, with a high probability, one can suppose that the drift losses of the trapped fusion $\alpha$ particles were measured in these experiments.

A decay-time both for ${ }^{3} \mathrm{He}$-ions and lost fusion alphas were measured during notches in the ICRH power when the accelerated ion source was strongly reduced or switched off at all. Decay times of intensities of 3.4-MeV $\alpha$-particle losses $\left(\tau_{\mathrm{L}}\right)$ and $\gamma$ rays $\left(\tau_{\gamma}\right)$ from ${ }^{3} \mathrm{He}$-reactions were derived. It is found that $\tau_{\gamma}>\tau_{\mathrm{L}}$ that is consistent with guess that change of the losses are faster than fusion $\alpha$-particle source strength.

\section{Observation of MHD effects}

In JET experiments on fast $H$-ion redistribution carried out with low-density plasmas and high ICRH power, large populations of highly energetic fast ions were built up and a variety of instabilities were destabilised [11,12]. Two types of fast ions were identified by the $\gamma$-ray diagnostics: fast $H$-ions with an energetic tail in excess of 4.5 MeV due to ICRH tuned to the fundamental H-resonance $\left(\omega=\omega_{\mathrm{H}}\right)$ in the centre of the plasmas and $D$-ions accelerated with second harmonic heating whose energies 
exceeded $0.5 \mathrm{MeV}$. Fast ion losses associated to MHD activity were detected with scintillator probe. Three types of losses were identified, one associated with sawtooth crashes, one associated with TAE and high frequency fishbones and a third group that seems to correlate with tornado modes. When tornado modes became unstable, a significant increase in the number of losses was measured and a change in both the energy and pitch angle of the lost ions was observed. In addition, it was also observed that the number of losses increased extensively with the ICRH power.

Most of JET pulses with long period (typically fast particle stabilised) sawtooth activity show a strong correlation between the time when particle losses are detected by the scintillator probe and start of the sawtooth crash.

\section{POSSIBLE IMPLEMENTATION OF $\gamma$-DIAGNOSTICS IN ITER}

In ITER the $\gamma$-ray diagnostics could perform the same functions as on JET. The main difference is a severe neutron/gamma background that requires special measures to maximise the signal-to-background noise ratio. For the fusion $\alpha$-particle measurements the 2-D $\gamma$-ray cameras could be used, however, the $\gamma$-ray detectors should be protected against harsh neutron emission with special neutron attenuators. The feasibility of the $\gamma$-ray measurements depends on effective the neutron suppression. It is important to avoid carbon-containing materials in the neutron attenuator because inelastic scattering of neutrons with energy exceeding $5 \mathrm{MeV}$, ${ }^{12} C\left(n, n^{\prime} \gamma\right)^{12} C$, leads to the unwelcome background of $4.44-\mathrm{MeV} \gamma$ rays. A more convenient neutron attenuator is ${ }^{6} \mathrm{LiH}$. It is compact, effective and rather transparent for $\gamma$-rays. It does not produce interfering $\gamma$-rays in the high-energy range. A $30-\mathrm{cm}$ sample of the ${ }^{6} \mathrm{LiH}$-filter reduces $2.4-\mathrm{MeV}$ neutron flux $\sim 900$ times and the $15-\mathrm{MeV}$ neutron flux $\sim 30$ times [13].

First experiments dedicated to the using ${ }^{6} \mathrm{LiH}$ for the $\gamma$-ray background reduction by means of neutron flux suppression have been carried out [14]. The test took place during JET experiments with $D D$-plasmas. A sample of the neutron attenuator ( $\varnothing 30 \mathrm{~mm} \times 300 \mathrm{~mm}$ ) was installed in the $2-\mathrm{m}$ vertical line-of-sight collimator used to view the plasma from above for fusion $\gamma$-ray spectrometry. Gamma-ray spectra recorded both with $\mathrm{BGO}$ - and $\mathrm{NaI}(\mathrm{Tl})$-detectors were used for analysis of the $\gamma$-ray background. It was found that $\gamma$-ray background is mainly induced by neutrons due to radiative capture, $(n, \gamma)$ and inelastic-scattering reactions, $\left(n, n^{\prime} \gamma\right)$. This neutron-induced gamma-background is produced in a) tokamak construction materials and b) detector itself. Only the last source of the background could be reduced with ${ }^{6} \mathrm{LiH}$-attenuator plugged in the collimator. Assessments of the reduction obtained from change intensity of $\gamma$-ray lines give a factor of 100. It is found that this type of $\gamma$-ray background lies in the energy range below $3 \mathrm{MeV}$. A small reduction (factor of 2) of the spectra was found in the energy range above $3 \mathrm{MeV}$, which is defined by $\gamma$-ray transparency of the ${ }^{6} \mathrm{LiH}$-attenuator. JET experiments with ${ }^{6} \mathrm{LiH}$ show that the attenuator is very effective in reduction of $\gamma$-ray background caused by $D D$-fusion neutrons, and results are consistent with neutron-generator measurements. Further tests in DT-discharges are needed to prove the full capability of ${ }^{6} \mathrm{LiH}$-attenuators as an 
indispensable component of the $\gamma$-ray diagnostic system in ITER. A sophisticated neutron attenuation system is being developed for the $\gamma$-ray cameras to allow the 2-D $\gamma$-ray measurement in the high performance JET discharges. Severe design constraints limited the attainable parameters of the upgraded $\gamma$-ray cameras, and a solution based on the use of pure light water was developed for the neutron attenuators. Lithium hydride (natural isotopic composition) will be used in another upgraded diagnostics: vertical and tangential $\gamma$-ray spectrometers.

If the main impurity in ITER plasmas is beryllium, the slowed down deuterons will give rise to gammas, $2.87 \mathrm{MeV}\left({ }^{10} B\right)$ and $3.37 \mathrm{MeV}\left({ }^{10} B e\right)$ from the ${ }^{9} B e(d, n \gamma){ }^{10} B$ and ${ }^{9} \mathrm{Be}(d, p \gamma){ }^{10} \mathrm{Be}$ reactions, whereas the fusion alphas with energies around the $2-\mathrm{MeV}$ resonance in the ${ }^{9} \mathrm{Be}(\alpha, n \gamma){ }^{12} \mathrm{C}$ reaction with produce $4.44-\mathrm{MeV}$ gammas. Furthermore, the source of fusion $\alpha$-particles can be obtained by measuring the $17-\mathrm{MeV}$ gammas from the $D(T, \gamma)^{5} \mathrm{He}$ reaction, which is a $10^{-5}$ branch of the $D(T, n)^{4} \mathrm{He}$ reaction. Using $\gamma$-ray spectrometers in every channel of the ITER neutron cameras, $\alpha$-particle slowing down profiles could be measured with the technique successfully tested in JET Trace Tritium Experiments [4]. Simultaneous measurements of the NBI power deposition and $\alpha$-particle slowing down profiles are very important for the optimisation of different plasma scenarios and understanding of the fast-ion confinement physics.

\section{ACKNOWLEDGMENTS}

This work was funded partly by the United Kingdom Engineering and Physical Sciences Research Council and by the European Communities under the contract of Association between EURATOM and UKAEA. Also this work was supported in part by US DoE Contract DE-AC02-76-CH03073 and government of the Russian Federation. The views and opinions expressed herein do not necessarily reflect those of the European Commission. This work was carried out within the framework of the European Fusion Development Agreement.

\section{REFERENCES}

1. G. J. Sadler et al Fusion Technology 18 (1990) 556.

2. O. N. Jarvis et al Nucl. Fusion 36 (1996) 1513.

3. V.G. Kiptily et al Nuclear Fusion 42 (2002) 999.

4. V.G. Kiptily et al Phys. Rev. Letters 93 (2004) 115001

5. V.G. Kiptily et al Nuclear Fusion 45 (2005) L21.

6. J. M. Adams et al Nucl. Instrum. Methods Phys. Res. A239 (1993) 277.

7. M.J. Mantsinen et al., Phys. Rev. Letters 88 (2002) 105002.

8. D. Darrow et al. Rev. Sci. Instrum. 77 (2006) 10E701

9. V.G. Kiptily et al., 33 EPS Conf. on Plasma Phys., Rome, 19 - 23 June 2006, P1.077.

10. L.C. Ingesson et al., Nucl. Fusion 38 (1998)1675

11. M. Reich et al., 34 EPS Conf. on Plasma Phys., Warsaw, July 2-6, 2007, O-4.016.

12. F. Nabais et al., 34 EPS Conf. on Plasma Phys., Warsaw, July 2-6, 2007, P-1.123

13. V.G. Kiptily et al., Technical Physics 43 (1998) 471

14. I.N. Chugunov et al. to be published in Instruments and Experimental Techniques (Pribory i Tekhnika Eksperimenta, in Russian) 